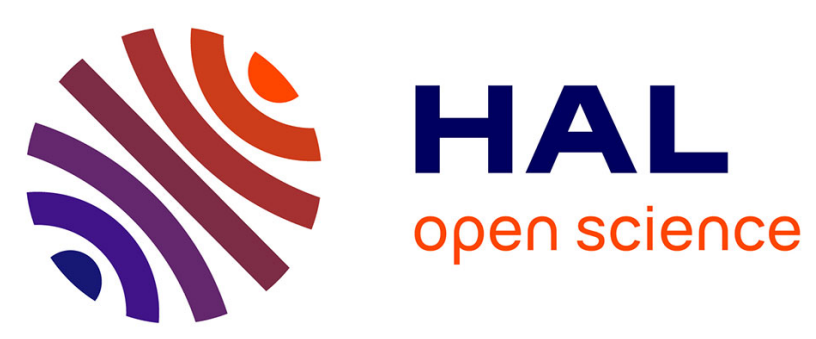

\title{
Theoretical modelling and experimental study of the fatigue of elastomers under cyclic loadings of variable amplitude
}

Audrey Jardin, Jean-Baptiste Leblond, Daniel Berghezan, Maude Portigliatti

\section{- To cite this version:}

Audrey Jardin, Jean-Baptiste Leblond, Daniel Berghezan, Maude Portigliatti. Theoretical modelling and experimental study of the fatigue of elastomers under cyclic loadings of variable amplitude. Comptes Rendus Mécanique, 2014, 342 (8), pp.450 - 458. 10.1016/j.crme.2014.04.002 . hal-01433992

\section{HAL Id: hal-01433992 \\ https://hal.sorbonne-universite.fr/hal-01433992}

Submitted on 13 Jan 2017

HAL is a multi-disciplinary open access archive for the deposit and dissemination of scientific research documents, whether they are published or not. The documents may come from teaching and research institutions in France or abroad, or from public or private research centers.
L'archive ouverte pluridisciplinaire HAL, est destinée au dépôt et à la diffusion de documents scientifiques de niveau recherche, publiés ou non, émanant des établissements d'enseignement et de recherche français ou étrangers, des laboratoires publics ou privés. 


\title{
Theoretical modelling and experimental study of the fatigue of elastomers under cyclic loadings of variable amplitude
}

\author{
A. $\operatorname{Jardin}^{\text {a }}$, J.B. Leblond ${ }^{\mathrm{a}}$, D. Berghezan ${ }^{\mathrm{b}}$, M. Portigliatti ${ }^{\mathrm{b}}$ \\ ${ }^{a}$ UPMC Univ Paris 06, CNRS, UMR 7190, Institut Jean Le Rond d'Alembert, Tour 65-55, 4 place Jussieu, 75252 Paris \\ Cedex 05, France \\ ${ }^{\mathrm{b}}$ Michelin, Centre de Recherches et de Technologies de Ladoux, rue Bleue, ZI Ladoux, 63118 Cebazat, France
}

Received $* * * * *$; accepted after revision +++++

\begin{abstract}
Deviations from Miner's linear law of cumulative damage have been modelled and observed many times for the fatigue of metals, but almost no analogous studies have been performed for elastomers. Such a study is reported here.

A simple phenomenological model, applicable to any type of material and able to quantitatively reproduce such deviations, is presented first. This model is based on continuum damage mechanics. It relates the fatigue damage of the material to the number of cycles through some suitable evolution law, in which the derivative of damage is expressed as a non-factorizable function of the instantaneous load cycle and the damage itself.

Fatigue experiments performed on "diabolo" specimens made of two different elastomeric materials and subjected to two successive cyclic loads of different amplitudes are then reported. Significant deviations from Miner's rule are observed: Miner's "total cumulated damage" may be lower or larger than unity by a small or large amount, depending on the sequence of loadings and the type of material. As a rule, the deviation from Miner's rule systematically changes sign upon reversal of the sequence of loadings. The model is shown to allow for an acceptable reproduction of the experimental results, and especially of this systematic change of sign.

To cite this article: J.B. Leblond, H.A. El Sayed, J.M. Bergheau, C. R. Mecanique? (2014).
\end{abstract}

Key words: Elastomers; fatigue; deviations from Miner's rule; continuum damage model; non-factorizable evolution equation

\section{Introduction}

Rubber components often experience complex cyclic loadings involving 3D stress states varying arbitrarily in time. Prediction of fatigue under such general conditions is important for the prediction of the durability of these components.

Models for the fatigue of elastomers under general cyclic loadings have been reviewed by Mars and Fatemi [1], who distinguished between models based on consideration of crack initiation and propagation, respectively. We shall focus here on the first class of models, initiated by Cadwell et al. [2]), since propagation-based models are applicable only when the location and size of the initial crack(s) are known, which is seldom the case in practice.

Email addresses: jbl@lmm.jussieu.fr (J.B. Leblond), daniel.berghezan@fr.michelin.com (D. Berghezan), maude.portigliatti@fr.michelin.com (M. Portigliatti). 
In a previous paper (Brunac et al. [3]), we considered the problem of predicting the fatigue lifetime under general 3D but perfectly time-periodic cyclic loadings. We now envisage the question of description of fatigue under cyclic loadings varying in time.

The standard answer to this problem consists of using Miner's [4] heuristic, but appealingly simple and elegant linear rule of cumulative damage. The applicability and limits of this rule have been assessed in numerous experimental works in the case of metals; as a rule, in such materials, Miner's "total cumulated damage" is observed to be smaller than unity if the more severe load cycles are applied first, and larger than unity if the less severe cycles are applied first (Chaboche and Lesne [5]). In contrast, very few similar studies have been performed for elastomers. The purpose of this paper is therefore to describe a theoretical and experimental investigation of deviations from Miner's rule for the fatigue of elastomers.

The paper is organized as follows:

- Section 2 first describes a heuristic model retaining the basic simplicity of Miner's rule, but accounting for possible deviations from its predictions while allowing for an arbitrary dependence of the number of cycles at failure upon the load cycle. This model belongs to the category of continuum damage models, as described in the book of Lemaitre and Chaboche [6], and relies on some evolution equation for the damage variable in which this variable and the instantaneous load cycle ${ }^{1}$ appear in a "nonfactorized" form. It involves a single adjustable parameter depending on the load cycle.

- Section 3 then explains how the load-cycle-dependent material parameter introduced in the model may be determined from experimental numbers of cycles at failure observed in successions of different cyclic loads.

- Section 4 presents the set of experiments performed. These experiments involve load histories consisting of two cyclic loads of different amplitudes applied in succession on "diabolo" specimens made of two distinct elastomeric materials. They evidence clear and significant deviations from Miner's rule in at least one of these materials.

- Finally Section 5 presents the application of the model to the experiments performed. Determination of the values of the model parameter for the two load cycles considered in these experiments is shown to allow for a much better reproduction of the experimental results than Miner's standard rule.

\section{Continuum-damage-based description of fatigue under varying cyclic loadings}

A number of models accounting for possible deviations from Miner's rule of cumulative damage have been proposed. An extensive overview of the literature was provided by Fatemi and Yang [7]; historical references include the works of Marko and Starkey [8], Manson [9], Bui-Quoc et al. [10] and Subramanyan [11], among others. We shall focus here on models based on continuum damage mechanics.

\subsection{Generalities}

The application of the phenomenological theory of continuum damage mechanics to the description of fatigue was explained in the book of Lemaitre and Chaboche [6], and a more recent review was provided by Desmorat [12]. In this approach, the initiation of a macroscopic fatigue crack in an elementary volume of material is considered to be due to the progressive degradation of this volume, described through some damage variable $D$ lying in the interval $(0,1)$. This variable reduces the specific free energy by the factor $1-D$ or some variant. ${ }^{2}$ It obeys an evolution equation in which the number of cycles $N$ plays the role of time. The simplest possible one reads

$$
\frac{d D}{d N}=\frac{1}{N_{f}}
$$

where $N_{f} \equiv N_{f}(\mathcal{C})$ denotes the number of cycles at failure of the elementary volume, depending on the current load cycle $\mathcal{C}^{3}$. This law reproduces Miner's rule since the damage after $N$ non-identical cycles

1. This expression refers to the sequence of loadings undergone by the material during the cycle; the number of this cycle is immaterial.

2. A factor of $1-D^{\alpha}$, with $\alpha>1$, was argued by Brunac [13] to be more appropriate in the case of elastomers, on the grounds that the elastic stiffness is experimentally found to remain almost constant during the major part of the degradation process and decrease significantly only at its very end.

3. That is, again, on the sequence of loadings undergone by the material during the cycle, not on the number of this cycle. 
(with different values of $N_{f}$ ) is

A more general form of the evolution law of $D$ is

$$
D=\int_{0}^{N} \frac{d N}{N_{f}}
$$

$$
\frac{d D}{d N}=f(D, \mathcal{C})
$$

for some non-negative function $f$. When the load cycle $\mathcal{C}$ is invariable in time, integration of this evolution law yields

$$
\int_{0}^{D} \frac{d D^{\prime}}{f\left(D^{\prime}, \mathcal{C}\right)}=N
$$

It then follows from the definition of $N_{f}(\mathcal{C})$ as that number of cycles for which $D$ reaches unity under a succession of identical load cycles $\mathcal{C}$, that

$$
\int_{0}^{1} \frac{d D}{f(D, \mathcal{C})}=N_{f}(\mathcal{C})
$$

this is a necessary condition on the function $f$, which must hold for all possible load cycles $\mathcal{C}$.

A remarkable feature of the evolution equation (1) is the following equivalence:

$$
\text { (Miner's rule holds) } \Leftrightarrow f(D, \mathcal{C}) \text { is a "factorized" function of the form } \varphi(D) \psi(C) \text {. }
$$

Establishing the direct implication is more difficult than it may seem at first sight; a nice proof, briefly recalled in Appendix A for completeness, was provided by Stigh [14]. The proof of the converse implication is easier; see e.g. Lemaître and Chaboche [6].

One drawback of an evolution equation of type (1), in this form, is that it inevitably places restrictions upon the way the number of cycles at failure, $N_{f}$, depends upon the load cycle, $\mathcal{C}$. Indeed it is necessary in practice to ascribe some more or less complex analytical form to the function $f$; a typical example, in the case of metals, is provided by Chaboche and Lesne's [5] model. This is annoying because at least in the case of elastomers, the dependence of $N_{f}$ upon $\mathcal{C}$ is quite complex and not easily amenable to some analytic formula. (This is true even for $1 \mathrm{D}$ loadings, when the minimum stress reached during each cycle is nonzero).

For this reason, we shall henceforward consider evolution equations of type (1), but written in the form

$$
\frac{d D}{d N}=\frac{g\left(D, N_{f}\right)}{N_{f}} \quad, \quad N_{f} \equiv N_{f}(\mathcal{C})
$$

for some non-negative function $g$. The advantage of thus incorporating the dependence of the rate of damage upon the load cycle in an implicit form, through the number of cycles at failure, is that any dependence of $N_{f}$ upon $\mathcal{C}$ then becomes possible. It is not even necessary to assume any analytic form of dependence; the function $N_{f}(\mathcal{C})$ may be directly extracted from experiments. For 1D loadings for instance, it may be taken from Haigh's diagram which provides the experimental number of cycles at failure as a function of the maximum and minimum stresses reached during each cycle. For 3D loadings, it may be deduced from any model providing $N_{f}$ as a function of some characteristic elements of the load cycle; one may use for instance Brunac et al.'s [3] proposed heuristic extension of Haigh's diagram to completely arbitrary load cycles, inspired from Dang Van's [15] work on multiaxial fatigue of metals.

With the new form (4) of the evolution equation of $D$, the necessary condition (2) takes the form

$$
\int_{0}^{1} \frac{d D}{g\left(D, N_{f}\right)}=1
$$

for all possible load cycles $\mathcal{C}$. Also, property (3) takes the form

$$
\text { (Miner's rule holds) } \Leftrightarrow g\left(D, N_{f}\right) \text { is a factorized function. }
$$

\subsection{The model proposed}

We wish to define the simplest possible evolution equation of type (4) predicting deviations from Miner's rule, that is involving some non-factorized function $g\left(D, N_{f}\right)$. In view of the necessary condition (5), the 
simplest option is to consider a function $g\left(D, N_{f}\right)$ possessing the property that for every value of $N_{f}$, its inverse varies linearly with $D$ and takes the value 1 for $D=1 / 2$. This function is of the form

$$
g\left(D, N_{f}\right) \equiv \frac{1}{1+2 \beta(D-1 / 2)}=\frac{1}{1-\beta+2 \beta D} \quad, \quad \beta \equiv \beta\left(N_{f}\right) .
$$

Conditions (5) being then automatically satisfied, the only constraints on the possible values of the coefficient $\beta$ are those arising from the necessary non-negativeness of the function $g$ for $0 \leq D \leq 1$, which read

$$
-1 \leq \beta\left(N_{f}\right) \leq 1
$$

for all values of $N_{f}$.

Note that the function $g$ is non-factorized so that, by equation (6), deviations from Miner's rule are predicted as soon as the function $\beta\left(N_{f}\right)$ is not a constant.

Several evolution laws of type (1) involving non-factorized functions $f(D, C)$ have been proposed, notably by Chaboche and Lesne [5]. These laws generally included special functions $f$ in analytical form, and therefore did not allow for an arbitrary dependence of the number of cycles at failure upon the load cycle. One interesting exception, however, is the law proposed in the book of Chaboche [16]:

$$
\frac{d D}{d N}=\frac{D^{\alpha}}{(1-\alpha) N_{f}} \quad, \quad \alpha \equiv \alpha(\mathcal{C}) \quad, \quad N_{f} \equiv N_{f}(\mathcal{C}),
$$

with $\alpha<1$ so as to ensure convergence of the integral (2) defining $N_{f}$. This evolution equation possesses the same basic features as that defined by equations (4) and (7) since it allows for an arbitrary dependence of $N_{f}$ upon $\mathcal{C}$, while predicting deviations from Miner's rule as soon as the function $\alpha(\mathcal{C})$ is not a constant. The difference with the model proposed here lies in the form of the function $g$, the inverse of which is a power function of $D$ instead of a linear one. Some consequences of this difference are as follows:

- Chaboche's form (9) of the evolution equation distinguishes between the beginning and the end of the fatigue process (since the factor $D^{\alpha}$ particularizes the beginning), whereas formula (7) does not.

- In Chaboche's model, the initial damage rate can take only three possible values, $+\infty, 1 / N_{f}$ and 0 , depending on whether $\alpha$ is negative, zero or positive, whereas all values from $1 /\left(2 N_{f}\right)$ to $+\infty$ are possible in the model proposed here.

\section{Determination of model parameters from experiments}

Consider a fatigue experiment consisting of a succession of $N_{1}$ load cycles $\mathcal{C}_{1}$ followed by $N_{2}$ load cycles $\mathcal{C}_{2}$, leading to failure. Assume that the evolution of damage is governed by equations (4) and (7). During the first phase, equation (4) reads $d D / d N=g\left(D, N_{f 1}\right) / N_{f 1}$ where $N_{f 1} \equiv N_{f}\left(\mathcal{C}_{1}\right)$. It follows through integration that the damage $\mathcal{D}$ at the end of this phase is given by the equation

$$
G\left(\mathcal{D}, N_{f 1}\right)=\frac{N_{1}}{N_{f 1}} \quad, \quad G\left(D, N_{f}\right) \equiv \int_{0}^{D} \frac{d D^{\prime}}{g\left(D^{\prime}, N_{f}\right)} \equiv\left[1-\beta\left(N_{f}\right)\right] D+\beta\left(N_{f}\right) D^{2} .
$$

During the second phase, the evolution equation becomes $d D / d N=g\left(D, N_{f 2}\right) / N_{f 2}$ where $N_{f 2} \equiv N_{f}\left(\mathcal{C}_{2}\right)$. It again follows through integration that

$$
\int_{\mathcal{D}}^{1} \frac{d D}{g\left(D, N_{f 2}\right)}=1-G\left(\mathcal{D}, N_{f 2}\right)=\frac{N_{2}}{N_{f 2}}
$$

where equation (5) has been used.

Equations (10) and (11) provide two relations connecting the unknown coefficients $\beta_{1} \equiv \beta\left(N_{f 1}\right)$, $\beta_{2} \equiv \beta\left(N_{f 2}\right)$, the unknown damage parameter $\mathcal{D}$ at the end of the first phase and the two experimentally known "damage parameters in the sense of Miner", or "Miner damages"

$$
\left\{\begin{array}{l}
D_{1}^{\text {Miner }} \equiv N_{1} / N_{f 1} \\
D_{2}^{\text {Miner }} \equiv N_{2} / N_{f 2} .
\end{array}\right.
$$

To eliminate the undesired quantity $\mathcal{D}$ between these equations, one may first eliminate $\mathcal{D}^{2}$ to get

$$
\mathcal{D}=\frac{\beta_{1}\left(1-D_{2}^{\mathrm{Miner}}\right)-\beta_{2} D_{1}^{\mathrm{Miner}}}{\beta_{1}-\beta_{2}}
$$


and then reinsert this value into the sum of equations $(10)_{1}$ and (11) to get

$$
\begin{aligned}
F\left(D_{1}^{\text {Miner }}, D_{2}^{\text {Miner }} ; \beta_{1}, \beta_{2}\right) \equiv & \frac{\left[\beta_{1}\left(1-D_{2}^{\text {Miner }}\right)-\beta_{2} D_{1}^{\text {Miner }}\right]^{2}}{\beta_{1}-\beta_{2}} \\
& -\beta_{1}\left(1-D_{2}^{\text {Miner }}\right)+\beta_{2} D_{1}^{\text {Miner }}+1-D_{1}^{\text {Miner }}-D_{2}^{\text {Miner }}=0 .
\end{aligned}
$$

This is an equation connecting the sole unknown coefficients $\beta_{1}, \beta_{2}$ to the known Miner damages $D_{1}^{\text {Miner }}$, $D_{2}^{\text {Miner }}$.

Equation (14) does not, of course, suffice to determine $\beta_{1}$ and $\beta_{2}$. However, assume that another fatigue experiment consisting of a succession of $N_{2}^{\prime}$ load cycles $\mathcal{C}_{2}$ followed by $N_{1}^{\prime}$ load cycles $\mathcal{C}_{1}$, again leading to failure, is available. The same reasoning as before leads to the new, symmetric relation

$$
\begin{aligned}
F\left(D_{2}^{\text {Miner ' }}, D_{1}^{\text {Miner ' }} ; \beta_{2}, \beta_{1}\right)= & \frac{\left[\beta_{2}\left(1-D_{1}^{\text {Miner ' }}\right)-\beta_{1} D_{2}^{\text {Miner } ~}\right]^{2}}{\beta_{2}-\beta_{1}} \\
& -\beta_{2}\left(1-D_{1}^{\text {Miner } \prime}\right)+\beta_{1} D_{2}^{\text {Miner ' }}+1-D_{2}^{\text {Miner ' }}-D_{1}^{\text {Miner ' }}=0
\end{aligned}
$$

where $D_{2}^{\text {Miner ' }} \equiv N_{2}^{\prime} / N_{f 2}, D_{1}^{\text {Miner ' }} \equiv N_{1}^{\prime} / N_{f 1}$ are the new Miner damages. Equations (14) and (15), put together, allow to determine coefficients $\beta_{1}$ and $\beta_{2}$. If more experiments are available, the set of equations on these coefficients becomes over-determined, but may be solved in a least-squares sense. In all cases it is necessary to check that the necessary conditions (8) are satisfied, and if not to replace $\beta_{1}$ or $\beta_{2}$ by the maximum or minimum value allowed.

Remark. Consider the same two experiments as above, with the same notations plus $\mathcal{D}^{\prime}$, which represents the damage at the end of the first phase of the second experiment. By equations $(10)_{1}$ and (11),

$$
\left\{\begin{array}{l}
D_{1}^{\text {Miner }}+D_{2}^{\text {Miner }}=1+G\left(\mathcal{D}, N_{f 1}\right)-G\left(\mathcal{D}, N_{f 2}\right) \\
D_{2}^{\text {Miner } ~}+D_{1}^{\text {Miner }}=1+G\left(\mathcal{D}^{\prime}, N_{f 2}\right)-G\left(\mathcal{D}^{\prime}, N_{f 1}\right) .
\end{array}\right.
$$

Now it is easy to check that for the specific function $G$ defined by equation $(10)_{2}$, the expression $G\left(\mathcal{D}, N_{f 1}\right)-G\left(\mathcal{D}, N_{f 2}\right)$, considered as a function of $\mathcal{D}$, has a constant sign; hence $G\left(\mathcal{D}, N_{f 1}\right)-G\left(\mathcal{D}, N_{f 2}\right)$ and $G\left(\mathcal{D}^{\prime}, N_{f 2}\right)-G\left(\mathcal{D}^{\prime}, N_{f 1}\right)$ are of opposite signs, whatever the values of $\mathcal{D}$ and $\mathcal{D}^{\prime}$. It follows that if

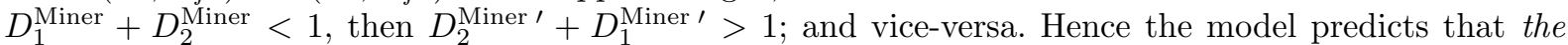
deviation from Miner's rule, measured by the quantity $D_{1}^{\mathrm{Miner}}+D_{2}^{\mathrm{Miner}}-1$, necessarily changes sign upon reversal of the sequence of loadings.

\section{Experimental procedure}

What follows is just a brief presentation of the experiments performed. More details are provided in Jardin's thesis [17].

\subsection{General presentation}

Fatigue experiments are performed on "diabolo" specimens. Figure 1 shows a photograph of one such specimen. The height and minimum diameter are $18 \mathrm{~mm}$ and $8 \mathrm{~mm}$ respectively. The specimens are made of two elastomeric materials, denoted A and B in the sequel, which differ through their carbon contents.

The specimen are subjected to various $1 \mathrm{D}$ cyclic loadings at room temperature. The frequency of the cycles is 3 or $6 \mathrm{~Hz}$, depending on the experiment; such low frequencies warrant negligible heating of the specimens. In each cyclic loading considered, the force applied varies between zero and some maximum value. Each experiment is performed at least three times on different specimens, in order to get an estimate of the experimental scatter.

The number of cycles at failure of the specimen is recorded for each experiment. This number is identified to that corresponding to initiation of a macroscopic crack in an elementary volume of material. The underlying hypothesis is that many more cycles are required for crack initiation than for crack propagation. Results shown below will be seen to support this approximation. Another argument is that it only leads to some systematic overestimation of the number of cycles necessary for initiation of a macroscopic crack, which should not affect the qualitative conclusions drawn. 


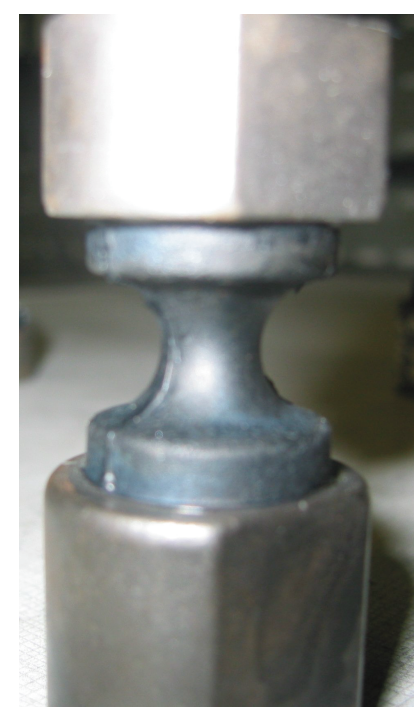

Figure 1. "Diabolo" specimen

\subsection{Determination of numbers of cycles at failure}

Prior to studying deviations from Miner's rule, it is necessary to determine the number of cycles at failure for a given cyclic load of constant amplitude. The loads considered are chosen so as to lead to complete failure within a "reasonable" number of cycles $N_{f}$. (Loads yielding very small values of $N_{f}$ are simply not acceptable, whereas those yielding very large values are uninteresting since they have no effect on the fatigue behavior). The maximum forces used are 60 and $90 \mathrm{~N}$ for material $\mathrm{A}$, and 110 and $130 \mathrm{~N}$ for material B.

Figure 2 shows the load-displacement curve during a typical test. The minimum force applied is not strictly zero but slightly negative, but this is harmless since negative stresses have no effect on the fatigue behavior. The maximum force applied can be seen to remain almost constant, as desired, even when the displacement increases considerably during propagation of the crack and final failure of the specimen. Also, this final phase can be observed to approximately extend from cycle 270,000 to cycle 300,000, which represents only $10 \%$ of the total number of cycles; this justifies the approximate identification of the numbers of cycles at initiation of a macroscopic crack and at failure of the specimen.

The numbers of cycles at failure found are as follows: for material A, 135,000 with a scatter of about $10 \%$ for a maximum force of $60 \mathrm{~N}$ and 20,000 with a scatter of about $35 \%$ for a maximum force of 90 $\mathrm{N}$; for material B, 300,000 with a scatter of about 30\% for a maximum force of $110 \mathrm{~N}$ and 37,000 with a scatter of about $5 \%$ for a maximum force of $130 \mathrm{~N}$.

\subsection{Experimental study of deviations from Miner's law}

Once the values of $N_{f}$ are known for the cyclic loads considered, deviations from Miner's law are studied by subjecting specimens to successions of two different cyclic loads. The effect of the sequence of loads is studied by systematically reverting it. Two possibilities are envisaged for each sequence: $0.33 N_{f}$ cycles of the first cyclic load, then enough cycles of the second to break the specimen; and similarly with $0.66 N_{f}$ cycles of the first cyclic load.

Figure 3 shows the load-displacement curve during a typical succession of cyclic loads. Again, the maximum force applied can be observed to remain almost constant during each of the cyclic loads applied in succession, as desired.

The numbers of cycles at failure thus determined experimentally are presented and compared to model predictions in Section 5 below. 


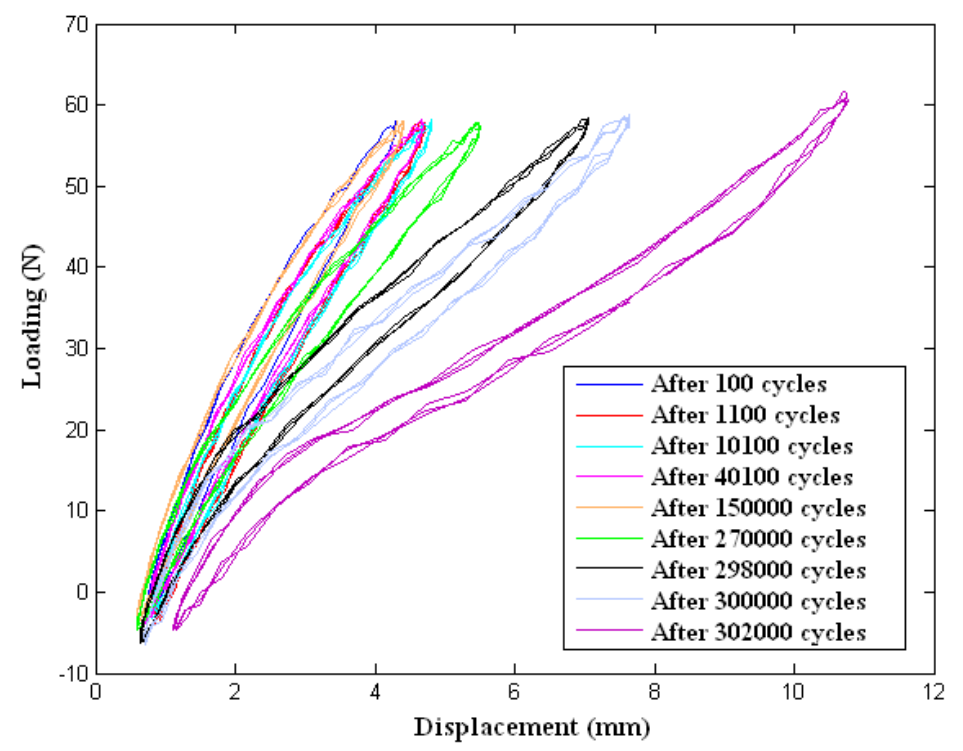

Figure 2. Load-displacement curve during a typical cyclic loading of constant amplitude

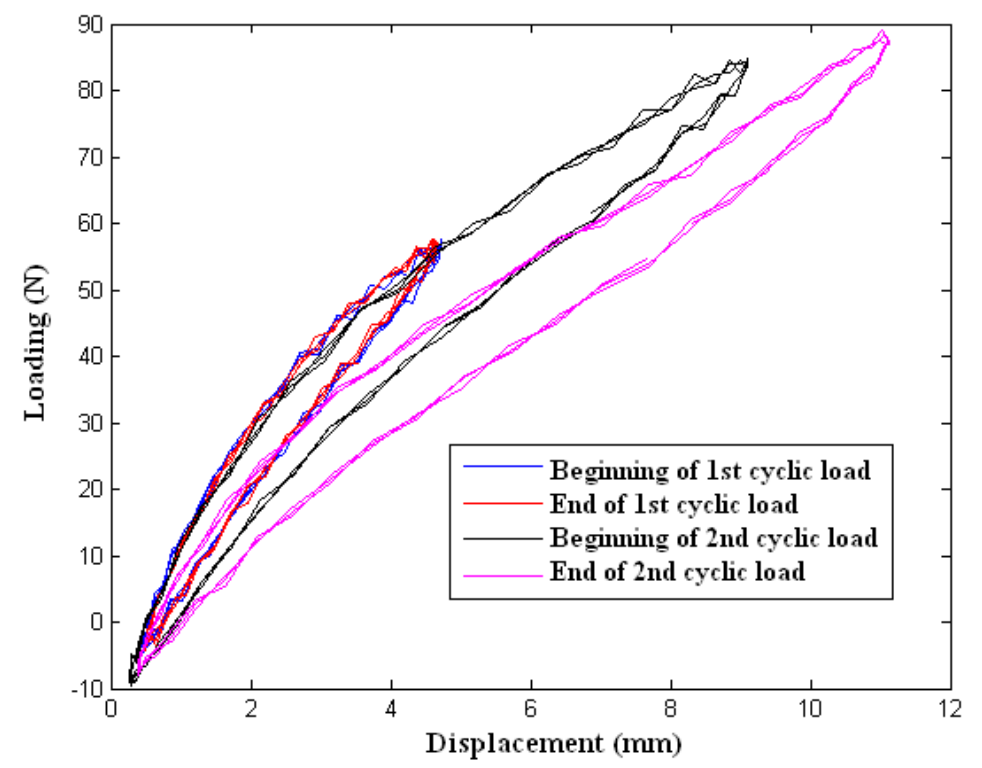

Figure 3. Load-displacement curve during a typical cyclic loading of variable amplitude

\section{Results and comparison with the model proposed}

Table 1 shows deviations from Miner's rule for material A. The quantities provided are the experimental and theoretical values of Miner's damages for the two phases of each experiment plus their sum, denoted as "Miner's total damage". There are 12 experiments, 6 for the loading sequence $\left(\mathcal{C}_{1}, \mathcal{C}_{2}\right)$ and 6 for the opposite one $\left(\mathcal{C}_{2}, \mathcal{C}_{1}\right) ; \mathcal{C}_{1}$ and $\mathcal{C}_{2}$ here denote the cyclic loads with maximum forces of $60 \mathrm{~N}$ and 90 $\mathrm{N}$ respectively. The model parameters $\beta_{1}$ and $\beta_{2}$ are determined through a least-squares fitting of the experimental results and amount to 0.437 and -0.340 respectively. Once this is done, one uses equation (13) with these values of $\beta_{1}$ and $\beta_{2}$ to calculate the damage $\mathcal{D}$ at the end of the first phase of the loading, then equations (10) and (11) to calculate the model values of the Miner damages of the two phases.

The model can be seen to reproduce the experimental results tolerably well. One interesting observation is the general experimental tendency toward a lower deviation from Miner's rule $\left(D_{1}^{\text {Miner }}+D_{2}^{\text {Miner }}<1\right)$ 


\begin{tabular}{|c|c|c|c|c|c|c|}
\hline \multirow[b]{2}{*}{ Load order } & \multicolumn{2}{|c|}{$D_{1}^{\text {Miner }}$} & \multicolumn{2}{|c|}{$D_{2}^{\text {Miner }}$} & \multirow{2}{*}{$\begin{array}{c}D^{\text {Miner }} \\
\text { total exp. }\end{array}$} & \multirow{2}{*}{$\begin{array}{c}D^{\text {Miner }} \\
\text { total pred. }\end{array}$} \\
\hline & $\begin{array}{c}D_{1}^{\text {Miner }} \\
\text { exp. }\end{array}$ & $\begin{array}{l}D_{1}^{\text {Miner }} \\
\text { pred. }\end{array}$ & $\begin{array}{c}D_{2}^{\text {Miner }} \\
\text { exp. }\end{array}$ & $\begin{array}{c}D_{2}^{\text {Miner }} \\
\text { pred. }\end{array}$ & & \\
\hline \multirow{6}{*}{$\left(\mathcal{C}_{1}, \mathcal{C}_{2}\right)$} & 0.33 & 0.17 & 0.81 & 0.70 & 1.14 & 0.87 \\
\hline & 0.33 & 0.44 & 0.31 & 0.38 & 0.64 & 0.82 \\
\hline & 0.33 & 0.55 & 0.13 & 0.28 & 0.46 & 0.83 \\
\hline & 0.66 & 0.80 & 0.01 & 0.11 & 0.67 & 0.91 \\
\hline & 0.66 & 0.50 & 0.44 & 0.32 & 1.10 & 0.82 \\
\hline & 0.66 & 0.64 & 0.23 & 0.22 & 0.89 & 0.86 \\
\hline \multirow{6}{*}{$\left(\mathcal{C}_{2}, \mathcal{C}_{1}\right)$} & 0.40 & 0.46 & 0.66 & 0.70 & 1.06 & 1.16 \\
\hline & 0.63 & 0.56 & 0.66 & 0.62 & 1.29 & 1.18 \\
\hline & 0.64 & 0.57 & 0.66 & 0.62 & 1.30 & 1.19 \\
\hline & 0.57 & 0.73 & 0.33 & 0.44 & 0.90 & 1.17 \\
\hline & 0.64 & 0.75 & 0.33 & 0.41 & 0.97 & 1.16 \\
\hline & 1.10 & 0.90 & 0.33 & 0.19 & 1.43 & 1.09 \\
\hline
\end{tabular}

Table 1

Comparison of experimental and predicted Miner damages for material A

\begin{tabular}{|c|c|c|c|c|c|c|}
\hline \multirow[b]{2}{*}{ Load order } & \multicolumn{2}{|c|}{$D_{1}^{\text {Miner }}$} & \multicolumn{2}{|c|}{$D_{2}^{\text {Miner }}$} & \multirow{2}{*}{$\begin{array}{c}D^{\text {Miner }} \\
\text { total exp. }\end{array}$} & \multirow{2}{*}{$\begin{array}{c}D^{\text {Miner }} \\
\text { total pred }\end{array}$} \\
\hline & $\begin{array}{c}D_{1}^{\text {Miner }} \\
\operatorname{exp.}\end{array}$ & $\begin{array}{c}D_{1}^{\text {Miner }} \\
\text { pred. }\end{array}$ & $\begin{array}{c}D_{2}^{\text {Miner }} \\
\text { exp. }\end{array}$ & $\begin{array}{c}D_{2}^{\text {Miner }} \\
\text { pred. }\end{array}$ & & \\
\hline \multirow{6}{*}{$\left(\mathcal{C}_{1}, \mathcal{C}_{2}\right)$} & 0.33 & 0.27 & 1.66 & 0.94 & 1.99 & 1.21 \\
\hline & 0.33 & 0.23 & 2.08 & 0.96 & 2.41 & 1.19 \\
\hline & 0.33 & 0.27 & 1.56 & 0.94 & 1.89 & 1.21 \\
\hline & 0.66 & 0.59 & 1.47 & 0.68 & 2.13 & 1.27 \\
\hline & 0.66 & 0.68 & 0.40 & 0.57 & 1.06 & 1.25 \\
\hline & 0.66 & 0.64 & 0.74 & 0.61 & 1.40 & 1.25 \\
\hline \multirow{6}{*}{$\left(\mathcal{C}_{2}, \mathcal{C}_{1}\right)$} & 0.13 & 0.13 & 0.66 & 0.74 & 0.79 & 0.87 \\
\hline & 0.06 & 0.07 & 0.66 & 0.86 & 0.72 & 0.93 \\
\hline & 0.05 & 0.06 & 0.66 & 0.87 & 0.71 & 0.93 \\
\hline & 0.15 & 0.18 & 0.33 & 0.65 & 0.48 & 0.83 \\
\hline & 0.11 & 0.14 & 0.33 & 0.71 & 0.44 & 0.85 \\
\hline & 0.08 & 0.12 & 0.33 & 0.76 & 0.41 & 0.88 \\
\hline
\end{tabular}

Table 2

Comparison of experimental and predicted Miner damages for material B

when the load with the smaller maximum force is applied first, and an upper deviation $\left(D_{1}^{\text {Miner }}+D_{2}^{\text {Miner }}>\right.$ 1) when the load with the larger maximum force is applied first. (There are some exceptions, but the deviation from Miner's rule is modest and probably not significant in these cases). Note that there is a difference with the case of metals here since as mentioned in the Introduction, for the latter materials, lower deviations from Miner's rule are systematically obtained by applying the larger load first.

Table 2 show results for material B in a similar way. The symbols $\mathcal{C}_{1}$ and $\mathcal{C}_{2}$ now refer to the cyclic loads with maximum forces of $110 \mathrm{~N}$ and $130 \mathrm{~N}$ respectively. The values of the model parameters $\beta_{1}$ and $\beta_{2}$ are -0.09 and 1 respectively; note that $\beta_{2}$ takes the maximum value allowed by inequality $(8)_{2}$. Experimental deviations from Miner's rule are notably larger than for material A. The reproduction of experimental results by the model is however still acceptable. It is interesting to note that a lower deviation is now obtained when the load with the larger maximum force is applied first, and an upper deviation when the load with the lower maximum force is applied first, in agreement with what is commonly observed in metals. The explanation of the difference of the behaviors of materials $\mathrm{A}$ and $\mathrm{B}$ in this respect is unknown. 
The following additional remarks are also in order:

- On average, the experiments on both materials confirm the model's prediction of a change of sign of the deviation from Miner's rule upon reversal of the sequence of loadings.

- In a few experiments (on material B), the Miner damage during the second phase of the loading exceeds unity. (In other words, the first cyclic loading seems to have paradoxically "reinforced" the material). The model is unfortunately unable to reproduce such a feature. Indeed it is clear from equation (11), where $G\left(\mathcal{D}, N_{f 2}\right)>0$, that $N_{2} / N_{f 2}$ cannot exceed unity. This restriction is not tied to the specific form (7) of the function $g$, but would subsist for any evolution equation of type (4).

\section{Conclusion}

A new, simple heuristic model for the fatigue of elastomers under variable cyclic loadings has been proposed. This model reproduces possible deviations from Miner's linear law of cumulative damage while allowing for an arbitrary dependence of the number of cycles at failure upon the instantaneous load cycle.

Experiments have been performed on "diabolo" specimens made of two different elastomeric materials and subjected to two different successive cyclic loads. These experiments evidence significant deviations from Miner's rule in at least one of the materials considered. The agreement between the experimental results and the model predictions, for suitable values of the adjustable material parameter involved, is acceptable. The experiments confirm in particular the model's prediction that the deviation from Miner's rule should change sign upon reversal of the sequence of loadings.

\section{References}

[1] W. Mars and A. Fatemi, A literature survey on fatigue analysis approaches for rubber, Int. J. Fatigue 24 (2002) 949-961.

[2] S. Cadwell, R.A. Merrill, C.M. Sloman and F.L. Yost, Dynamic fatigue life of rubber, Rubber Chem. Technol. 13 (1940) 304-315.

[3] J.B. Brunac, O. Gérardin and J.B. Leblond, On the heuristic extension of Haigh's diagram for the fatigue of elastomers to arbitrary loadings, Int. J. Fatigue 31 (2009) 859-867.

[4] M.A. Miner, Cumulative damage in fatigue, ASME J. Appl. Mech. 67 (1945) A159-A164.

[5] J.L. Chaboche and P.M. Lesne, A non-linear continuous fatigue damage model, Fatigue Fracture Engng. Mater. Structures 11 (1988) 1-17.

[6] J. Lemaître and J.L. Chaboche J.L., Mécanique des matériaux solides, Dunod (1996).

[7] A. Fatemi and L. Yang, Cumulative fatigue damage and life prediction theories: a survey of the state-of-the-art for homogeneous materials, Int. J. Fatigue 20 (1998) 9-34.

[8] S.M. Marko and W.L. Starkey, A concept of fatigue damage, Trans. ASME 76 (1954) 627-632.

[9] S.S. Manson, Interfaces between fatigue, creep, and fracture, Int. J. Fracture Mech. 2 (1966) 328-363.

[10] T. Bui-Quoc, J. Dubuc, A. Bazergui and A. Biron, Cumulative fatigue damage under stress-controlled conditions, ASME J. Basic Engng. 93 (1971) 691-698.

[11] S. Subramanyan, A cumulative damage rule based on the knee point of the SN curve, ASME J. Mater. Technol. 98 (1976) 316-321

[12] R. Desmorat, Damage and fatigue: continuum damage mechanics modeling for fatigue of materials and structures, Revue Européenne de Génie Civil 10 (2006) 849-877.

[13] J.B. Brunac, Modélisation numérique du comportement en fatigue des élastomères chargés de noir de carbone, Ph.D. thesis, Université Pierre et Marie Curie (Paris VI) (2006).

[14] U. Stigh, Continuum damage mechanics and life-fraction rule, ASME J. Appl. Mech. 73 (2006) 702-704.

[15] K. Dang Van, Introduction to fatigue analysis in mechanical design by the multiscale approach, CISM Courses and Lectures $n^{\circ} 392$ : High cycle metal fatigue, from theory to applications, Dang Van and Papadopoulos, Eds., SpringerVerlag, pp. 57-88 (1999).

[16] J.L. Chaboche, Cumul des dommages, Chapter 8 in : La fatigue des matériaux et des structures IV, Bathias and Pineau, Eds., Hermès (2009).

[17] A. Jardin, Modélisation et simulation numérique de la fatigue des élastomères soumis à des chargements cycliques complexes, Ph.D. thesis, Université Pierre et Marie Curie (Paris VI) (2009). 


\section{Appendix A. Appendix : Stigh's proof that Miner's rule implies factorization of the function $f(D, \mathcal{C})$}

Assume that fatigue is governed by the evolution law (1) of the damage variable $D$, and consider a loading history consisting of a succession of $N_{1}$ load cycles $\mathcal{C}_{1}$ followed by $N_{2}$ load cycles $\mathcal{C}_{2}$, leading to failure. During the first phase of this loading history, $D$ increases from 0 to some value $\mathcal{D}$, following the evolution equation $d D / d N=f\left(D, \mathcal{C}_{1}\right)$. Therefore

$$
N_{1}=\int_{0}^{\mathcal{D}} \frac{d D}{f\left(D, \mathcal{C}_{1}\right)} .
$$

Also, the number of cycles at failure for a succession of load cycles $\mathcal{C}_{1}$ is given by

$$
N_{f 1} \equiv N_{f}\left(\mathcal{C}_{1}\right)=\int_{0}^{1} \frac{d D}{f\left(D, \mathcal{C}_{1}\right)} .
$$

During the second phase of the loading history, $D$ further increases from $\mathcal{D}$ to 1 , following the evolution equation $d D / d N=f\left(D, \mathcal{C}_{2}\right)$. Therefore

$$
N_{2}=\int_{\mathcal{D}}^{1} \frac{d D}{f\left(D, \mathcal{C}_{2}\right)}=N_{f 2}-\int_{0}^{\mathcal{D}} \frac{d D}{f\left(D, \mathcal{C}_{2}\right)}
$$

where

$$
N_{f 2} \equiv N_{f}\left(\mathcal{C}_{2}\right)=\int_{0}^{1} \frac{d D}{f\left(D, \mathcal{C}_{2}\right)} .
$$

It follows from equations (A.1) - (A.4) that

and therefore that

$$
\frac{N_{1}}{N_{f 1}}+\frac{N_{2}}{N_{f 2}}=1+\frac{\int_{0}^{\mathcal{D}} \frac{d D}{f\left(D, \mathcal{C}_{1}\right)}}{\int_{0}^{1} \frac{d D}{f\left(D, \mathcal{C}_{1}\right)}}-\frac{\int_{0}^{\mathcal{D}} \frac{d D}{f\left(D, \mathcal{C}_{2}\right)}}{\int_{0}^{1} \frac{d D}{f\left(D, \mathcal{C}_{2}\right)}}
$$

$$
\text { (Miner's rule holds) } \Rightarrow \frac{N_{1}}{N_{f 1}}+\frac{N_{2}}{N_{f 2}}=1 \Rightarrow \frac{\int_{0}^{\mathcal{D}} \frac{d D}{f\left(D, \mathcal{C}_{1}\right)}}{\int_{0}^{1} \frac{d D}{f\left(D, \mathcal{C}_{1}\right)}}=\frac{\int_{0}^{\mathcal{D}} \frac{d D}{f\left(D, \mathcal{C}_{2}\right)}}{\int_{0}^{1} \frac{d D}{f\left(D, \mathcal{C}_{2}\right)}}
$$

for all load cycles $\mathcal{C}_{1}$ and $\mathcal{C}_{2}$. By equation (A.5), if Miner's rule applies, the function $\int_{0}^{D} \frac{d D^{\prime}}{f\left(D^{\prime}, \mathcal{C}\right)} / \int_{0}^{1} \frac{d D^{\prime}}{f\left(D^{\prime}, \mathcal{C}\right)}$ is independent of the load cycle $\mathcal{C}$. Denote this function $\Phi(D)$. Then

Differentiating this equation with respect to $D$, one gets

$$
\int_{0}^{D} \frac{d D^{\prime}}{f\left(D^{\prime}, \mathcal{C}\right)} \equiv \Phi(D) \Psi(\mathcal{C}) \quad, \quad \Psi(\mathcal{C}) \equiv \int_{0}^{1} \frac{d D^{\prime}}{f\left(D^{\prime}, \mathcal{C}\right)}
$$

$$
\frac{1}{f(D, \mathcal{C})}=\Phi^{\prime}(D) \Psi(\mathcal{C}) \Rightarrow f(D, \mathcal{C})=\frac{1}{\Phi^{\prime}(D)} \frac{1}{\Psi(\mathcal{C})}
$$

which shows that the function $f(D, \mathcal{C})$ is factorized and concludes the proof. 